\title{
DISEÑO DE REDES DE LOGÍSTICA INVERSA: UNA REVISIÓN DEL ESTADO DEL ARTE Y APLICACIÓN PRÁCTICA
}

\author{
A NETWORK DESIGN FOR A REVERSE LOGISTICS: A REVIEW AND A PRACTICAL APPLICATION \\ Luz Ángela Flórez Calderón \\ Ing. Industrial, Investigadora, Universidad Tecnológica de Pereira, Pereira, Colombia \\ laflorez@utp.edu.co
}

Eliana Mirledy Toro Ocampo

Ing. Industrial, M.Sc., Profesor asociado, Facultad de ingeniería industrial.

Universidad Tecnológica de Pereira, Pereira, Colombia

elianam@utp.edu.co

Mauricio Granada Echeverry

Ing. Eléctrico, M.Sc., Ph.D. Profesor asociado, Facultad de Ingeniería Eléctrica,

Universidad Tecnológica de Pereira, Pereira, Colombia

magra@utp.edu.co

Fecha de recepción: 28 de abril de 2012

Fecha de aprobación: 10 de diciembre de 2012

\section{RESUMEN}

Este artículo presenta una revisión de diferentes modelos de programación matemática y técnicas de solución, aplicadas en la solución de problemas de diseño de redes de logística inversa, en donde se describen las principales contribuciones y se comparan varios tratamientos, modelos y algoritmos de solución. Adicionalmente, se ha desarrollado una aplicación al caso específico de la gestión de llantas fuera de uso en las ciudades de Pereira y Dosquebradas (Colombia), evaluando las alternativas que existen en la recuperación y valorización de este tipo de residuo, con el fin de incurrir en el menor costo posible y/o generar beneficios para la Región.

Palabras clave: logística inversa, modelos de programación matemática, técnicas de solución, recuperación y valorización, residuos.

\begin{abstract}
This paper offers a review of several math programming models and resolution techniques applied to solve design issues of reverse logistic networks, which describes the key contributions and compares several approaches, models and solution algorithms. Additionally, an application
\end{abstract}


has been developed for a specific management of disused tires in Pereira and Dosquebradas (Colombia), evaluating choices to recovery and assessment of such a waste in order to generate the lowest cost and/or produce benefits for that area.

Keywords: reverse logistics, math programming models, solution techniques, recovery and assessment, waste.

\section{INTRODUCCIÓN}

Los problemas presentados en el diseño de redes de logística inversa, se han centrado principalmente en la gestión de residuos, recuperación de materiales (reciclaje), recuperación de productos o de partes (remanufactura o reuso) y retornos comerciales [1], así como, en la redistribución de los bienes recuperados o nuevos que han sido elaborados a partir de los residuos.

Con el fin de diseñar herramientas que permitan apoyar la toma de decisiones, autores como Fleischmann et al [2] y Bloemhof-Ruwaard et al [3] realizaron los primeros análisis acerca de la implementación de la investigación de operaciones, en el análisis de cadenas de suministro, así como de las posibilidades de incorporar temas ambientales en la gestión de las mismas.

Por lo general se han usado modelos de programación lineal entera mixta (MILP), en la solución de problemas de localización de instalaciones. Sin embargo, publicaciones recientes han adelantado otro tipo de planteamientos, con modelos de programación entera mixta estocásticos [4], modelos de programación entera mixta multi-objetivo [5, 6] y modelos no lineales [7]. En la gestión de inventarios, se han encontrado modelos de programación estocástica, así como de programación no lineal, que involucran procesos de decisión de Markov, tamaños de lote óptimo y modelos de simulación, entre otros.

Esta revisión se encuentra limitada a las publicaciones en revistas científicas y centrada en las publicaciones recientes, comprendidas entre 1990 y 2011.

Este documento se ha desarrollado de la siguiente manera: en el capítulo 1, se hizo una exploración de los avances que ha tenido el concepto de red logística o cadena de suministro, hacia la integración de la gestión de la cadena, tanto en las funciones del flujo directo como en las funciones del flujo inverso. En el capítulo 2, se desarrolló una revisión de la literatura, y en el capítulo 3, se presenta un modelo de logística inversa aplicado al problema de la gestión de llantas fuera de uso, en las ciudades de Pereira y Dosquebradas (Colombia). 


\section{MARCO CONCEPTUAL DE REFERENCIA}

\subsection{RED LOGÍSTICA O CADENA DE SUMINISTRO INTEGRAL}

Como actividad empresarial, la logística ha encontrado su desarrollo, aplicándose principalmente a las funciones de suministro, almacenamiento, producción, distribución y consumo. En el período comprendido entre 1960 y 2000, han ocurrido cambios relevantes en los sistemas logísticos que han logrado la integración de las actividades logísticas. En la actualidad, la logística comprende la gestión de materiales y la distribución física, con el apoyo de las tecnologías de la información, las estrategias de marketing y la planeación estratégica de las actividades para hacer una gestión eficiente [8].

Sin embargo, en los últimos años la sociedad está demandando a las empresas, además de servicios eficientes, comportamientos medioambientales más positivos, derivados de la preocupación creciente del alto consumo de productos y su inadecuada eliminación.

Es así como la logística moderna se orienta hacia la integración de la cadena, considerando dos sentidos: en primera instancia, el flujo directo que comprende el flujo de bienes e información a través de proveedores, productores, distribuidores, y consumidores, considerando después el sentido inverso, es decir, la recuperación de materiales y productos al final de su vida útil, para reintroducirlos en la misma cadena o en una nueva [9].

Este enfoque ha dado lugar al concepto de logística inversa, tema que ha sido de gran interés en las últimas décadas y ha tenido un gran desarrollo, aunque su aplicación no ha sido tan evidente.

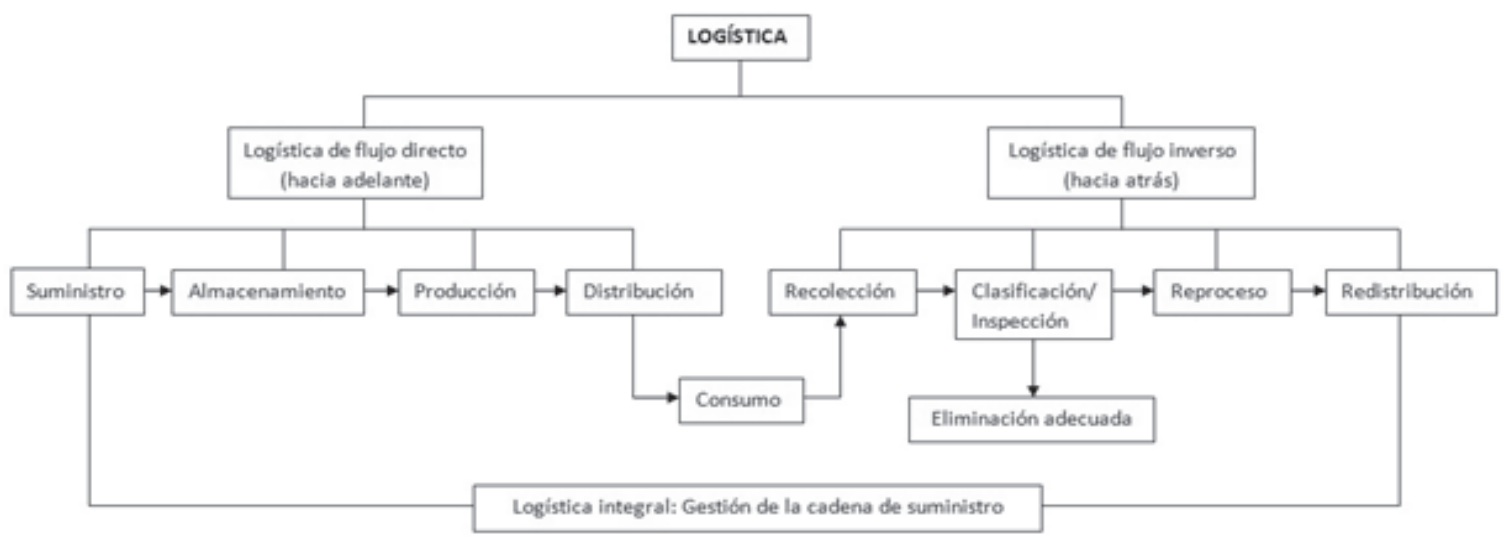

Figura 1. Red de logística integral

Fuente: Elaboración propia

La logística comprende entonces, la suma de variadas y complejas actividades, y a su vez, cada una de ellas se ha ocupado de extender el análisis, para profundizar en la creación de estratagemas que permitan el desempeño óptimo de redes logísticas o cadenas de suministro. En la Figura 1, 
se ilustran las actividades que comprenden la integración de la cadena de suministro, incluidas las actividades que comprende la logística inversa.

En algunas cadenas, es posible reintroducir el producto en la cadena inicial, dando lugar a ciclos cerrados (closed-loop supply chain); en otros casos, los ciclos son abiertos y dan lugar a nuevos mercados y cadenas de recuperación y valorización, donde se originan nuevas actividades de recolección, clasificación, reproceso y distribución.

Según lo señala Rubio S. [10], dos motivaciones importantes han dado lugar a la recuperación y aprovechamiento de productos fuera de uso o residuos generados: motivos legales y motivos económicos. Los motivos legales son consecuencia de las actuales regulaciones y normativas a la gestión de residuos y productos al final de su vida útil, estableciendo y señalando las obligaciones, y responsabilidades a los actores involucrados en la cadena, esto dependiendo claramente del contexto. Por su parte, los motivos económicos se refieren al interés de las empresas por obtener un valor añadido en la gestión adecuada de sus residuos o productos, ya sea mediante beneficios económicos o ventajas competitivas con estrategias de marketing y posicionamiento de la imagen de empresas medioambientalmente responsables.

Sin embargo, llevar a cabo las actividades logísticas para la recuperación y valorización de residuos y productos al final de su vida útil, requiere en algunos contextos, de complejos mecanismos de gestión y la articulación de un gran número de actores. Para esto, en países como España, se han creado sistemas integrados de gestión (SIG). EI SIG es una sociedad sin ánimo de lucro, donde participan los sectores interesados: productores, distribuidores, generadores, recuperadores y recicladores [1 1]. En este tipo de sistemas logísticos, existe un grado de cooperación mayor, dado que un único ente gestor se responsabiliza de la recuperación, recolección y gestión adecuada de los residuos.

\subsection{LOGÍSTICA INVERSA E INVESTIGACIÓN DE OPERACIONES}

Desde finales de los años 70, se empezaron a divulgar publicaciones en el tema de la logística inversa, debido al interés de muchos sectores sobre los beneficios potenciales de la recuperación de materiales. Autores como Rogers y Tibben-Lembke [9], liderados por el Council of Logistics Management, la editorial de los libros norteamericanos sobre logística inversa, han hecho importantes publicaciones. En Europa, se ha constituido la red europea sobre logística inversa: Rev Log (1998), en donde se han destacado importantes autores, por sus contribuciones en la solución de problemas presentados en la logística inversa.

La investigación de operaciones, como un enfoque científico en la toma de decisiones, busca operar un sistema por lo regular, en condiciones que requieren la asignación de recursos escasos [12]. Bajo esta metodología, se requiere el uso de un modelo matemático de optimización. Éste es una representación matemática de una situación real que se podría usar para tomar mejores decisiones.

LUZ ÁNGELA FLÓREZ CALDERÓN, ELIANA MIRLEDY TORO OCAMPO, MAURICIO GRANADA ECHEVERRY 
La gestión de residuos y recuperación de productos, es un problema complejo que requiere de planificación, gestión y control del flujo de materiales y productos [9], así como tomar decisiones a nivel estratégico y operativo [10]. Las decisiones de carácter estratégico, involucran problemas de localización de puntos de recolección de residuos y plantas de tratamiento, teniendo en cuenta las capacidades de procesamiento de las plantas y el almacenamiento de los puntos de recolección, el costo de apertura, los costos fijos y variables asociados en el proceso, la ubicación de los nuevos proyectos que utilizaran los residuos, su disponibilidad y demanda de residuos. Las decisiones de carácter operativo, se refieren a la cantidad y tamaño de las instalaciones de transporte, personal involucrado, problemas de enrutamiento asociado al flujo de materiales y gestión de inventario, entre otros.

Aunque las decisiones de carácter estratégico y operativo están interconectadas, el análisis a menudo se hace por separado, para simplificar. En consecuencia, se han generado diferentes tipos de clasificaciones, según los autores que han estudiado los problemas presentados en logística inversa, de acuerdo con el tipo de problema [2] o de acuerdo con la opción de recuperación [13].

\section{REVISIÓN DE LA LITERATURA EN LOGÍSTICA INVERSA: UNA COMPARACIÓN DE LOS MODELOS.}

Para comparar los modelos matemáticos, se clasificaron los artículos publicados, de acuerdo con el problema general que han tratado, tal como lo han presentado Fleischmann et al. [2], de la siguiente manera:

- Diseño de redes de logística inversa y localización de instalaciones

- Gestión de inventarios en flujos de retorno

- Planificación y control de actividades de producción

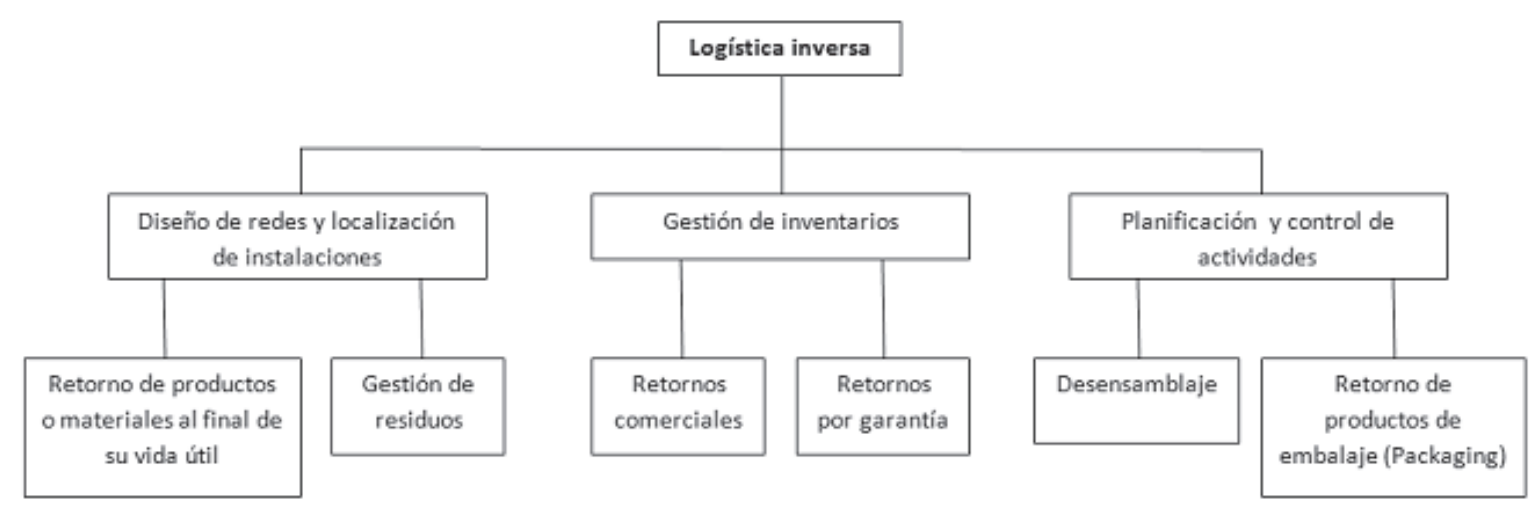

Figura 2. Problemas abordados desde logística inversa

Fuente: Elaboración propia 
En la Figura 2 se ilustran los problemas que han sido tratados en logística inversa, por diversos autores. Sin embargo, algunos contextos combinan decisiones de dos o más tipos de problemas. Es así como algunos autores han tratado problemas de localización de instalaciones, considerando retornos con incertidumbre, problemas de gestión de inventarios que involucran decisiones de transporte, así como tiempos de entrega.

\subsection{DISEÑO DE REDES DE LOGÍSTICA INVERSA Y LOCALIZACIÓN DE INSTALACIONES}

En primer lugar, se encuentran los artículos que tratan sobre la localización de instalaciones y los que tratan el diseño de redes de logística inversa. Este tipo de publicaciones se han centrado en la recolección de los productos o residuos y la redistribución de los bienes que se elaboran a partir de los mismos. Los aspectos fundamentales son el número y la localización de instalaciones para el tratamiento de residuos, el uso o no de puntos de recolección y la financiación de la red [1 1 1]. Este problema constituye la primera fase en la toma de decisiones para proponer una red de logística inversa.

Es así como las primeras aplicaciones formularon modelos de programación lineal entera mixta (MILP), que consideraban la capacidad posible de las instalaciones, y el planteamiento de escenarios para analizar otros factores, tales comola incertidumbre en el retorno de productos o los tipos de tecnología por implementar en la recuperación de los mismos. En esencia, la función objetivo (1) se formula como una función de costos definida como la sumatoria de los costos variables $\left(C_{i j}\right)$, de enviar las cantidades de productos o materiales $\left(\mathrm{X}_{\mathrm{ij}}\right)$, desde los puntos de recolección (i) hasta las plantas de tratamiento (j) y los costos fijos de apertura $\left(\mathrm{f}_{\mathrm{j}}\right)$ de las posibles plantas $\left(\mathrm{Y}_{\mathrm{j}}\right)$ :

$$
\min \sum_{i=1}^{M} \sum_{j=1}^{N} c_{i j} x_{i j}+\sum_{j=1}^{N} f_{j} y_{j}
$$

Es el caso de aplicaciones como la realizada por Spengler et al. [14], donde se formularon dos modelos con aplicaciones, por una parte al reciclaje y, por la otra, al desmantelamiento. En el primer modelo, se estudia el problema de localización de instalaciones y asignación de flujos. El modelo fue planteado como un problema de programación lineal entera mixta (MILP), con el objetivo de minimizar todos los costos asociados en el proceso de reciclaje. Se consideraron escenarios con diferentes tipos de tecnología por implementar en los procesos de recuperación. El segundo modelo consiste en un problema de maximización de una función de beneficios, y presenta dos fases:la planeación de las actividades de desmantelamiento y la planeación de las actividades de reciclaje. Fue planteado como un modelo de programación lineal entera mixta (MILP); sin embargo, dada la complejidad del modelo, los autores aplicaron el algoritmo de descomposición de Benders, y transformaron el modelo inicial en dos tipos de problema.

Otras aplicaciones similares han sido la de Barros et al. [15], que diseñan una red para la recuperación y el reciclaje de arena proveniente de construcciones. Este modelo de carácter estático, consideró distintos escenarios para tratar la incertidumbre en la cantidad y calidad de la arena recuperada.

LUZ ÁNGELA FLÓREZ CALDERÓN, ELIANA MIRLEDY TORO OCAMPO, MAURICIO GRANADA ECHEVERRY 
Por su parte, Du et al. [5], con un nuevo enfoque, plantean un modelo biobjetivo que busca minimizar los costos totalesy minimizar el retraso en el tiempo de ciclo para el diseño de una red inversa de un servicio de reparación posventa. Este tipo de modelo se preocupa por conocer el número de instalaciones que se requiere para disminuir el tiempo de atención a los clientes, y considera la capacidad de las plantas. Adiciona la consideración de economías de escala, con un incremento en los costos asociados a una unidad. El modelo busca encontrar soluciones no dominadas y es resuelto mediante una combinación de varios métodos: simples dual y búsqueda dispersa.

Para Cruz et al. [16], la función objetivo está en función de los costos generados y del porcentaje de cobertura. Ellos formularon en México, un modelo para la recolección de vehículos al final de su vida útil. Plantean tres posibles escenarios, en donde se recolecta el 100\%, $90 \%$ y 75\% respectivamente, del total de vehículos generados. El objetivo es conocer el número de instalaciones requeridas, así como su ubicación, minimizando los costos asociados. Los mismos puntos que generan vehículos, se convierten en potenciales puntos de localización de las instalaciones para la recolección. Como método de solución, utilizaron relajación langrangiana.

Srivastava S.K. [17], ha desarrollado un modelo conceptual para la recolección y el reciclaje de múltiples productos retornados, y en una segunda parte, ha probado maximizar beneficios para varios escenarios. Para desarrollar su propuesta, ha usado un modelo de optimización jerárquico de dos niveles, para dar una visión útil de la gestión y sus implicaciones. Esto le ha permitido reducir un poco el tamaño del problema para su análisis. Los escenarios se refirieron a cambios en la información de la cantidad y calidad de los productos retornados; sin embargo, no se tuvo en cuenta, restricciones de capacidad. Como herramienta de solución, implementaron el GAMS (general algebraic modeling system).

Fan Wang et al. [18], plantean un modelo general para el análisis de problemas de localización en redes de logística inversa cerradas. El modelo tiene en cuenta los niveles de capacidad de las instalaciones, en un único período. Consideran como método de solución, el árbol de expansión mínima con algoritmos genéticos.

Frota Neto et al. [19], estudian un modelo para la asignación de los flujos de productos retornados, considerando instalaciones fijas existentes, con el objetivo de minimizar el costo de las operaciones y el impacto ambiental. El modelo es un modelo de programación multi-objetivo que recurre al análisis de datos envolvente: data envelopment analysis (DEA), para la consideración del impacto ambiental. Por último, encuentra la frontera eficiente de Pareto, para encontrar una solución óptima.

De manera similar, Dehghanian et al. [6], con un análisis más completo, estudiaron un modelo de programación matemática multi-objetivo, para diseñar una red de recuperación sostenible, en donde el impacto económico, ambiental y social, es balanceado, con el fin generar una alternativa para una red de reciclaje de desechos de llantas. Por medio del análisis de ciclo de vida (LCA), se determinó el impacto ambiental de las diferentes opciones para la gestión de llantas fuera de 
uso por evaluar. Así mismo, se ha desarrollado un proceso de jerarquía analítica, para determinar el impacto social. El objetivo es maximizar los beneficios económicos y sociales y minimizar los impactos ambientales negativos, a la vez. En la red de recuperación de desechos de llantas, cada solución corresponde a diferentes configuraciones de la red, basados en el tipo de tecnología y la ubicación de las instalaciones de las plantas de reciclaje. El problema ha sido formulado mediante un modelo de programación multi-objetivo con algoritmos genéticos: Multi-objetive genetic algorithm (MOGA), para encontrar la solución óptima mediante la frontera de Pareto.

Ortega M. [11] por su parte, encuentra que los modelos han sido generalmente de carácter estático, por lo cual propone una metodología de solución para el problema de localización de instalaciones con múltiples posibilidades de capacidad y múltiples períodos, y lo ajusta al caso de recuperación del residuo ligero de fragmentación de los vehículos fuera de uso (con carácter dinámico). También resuelve el problema de localización de una planta de tratamiento y los centros de transferencia en una región determinada (con carácter dinámico y estocástico). Formula problemas de programación lineal entera mixta (MILP), y emplea técnicas meta-heurísticas en la solución: Algoritmos genéticos y búsqueda dispersa. Concluye con una comparación de los métodos de solución entre técnicas exactas, búsqueda dispersa y algoritmos genéticos, y encuentra más eficientes las técnicas exactas para problemas de menor tamaño, y búsqueda dispersa para la resolución de problemas mayores.

Hu et al. [20], plantearon un problema de localización de instalaciones, para la gestión de residuos peligrosos. El modelo propuesto considera múltiples períodos y diversas clases de residuos. Adicionalmente, consideran regulaciones gubernamentales. El modelo es un modelo lineal de tiempo discreto. Como método de solución, implementan LINDO (linear, interactive and discrete optimizer).

Lu et al. [21], consideran en un solo modelo, el flujo directo e inverso en una red logística, para actividades de remanufactura en productos retornados. Formulan un modelo de programación lineal entera mixta, donde las instalaciones son de tres tipos: productores, centros de remanufactura y centros intermedios. Introducen porcentajes para los flujos de productos. Como método de solución, implementan técnicas heurísticas: relajación langrangiana.

Algunos autores se centraron en modelos genéricos para redes de logística inversa. Yongsheng et al. [22], establecen un modelo general para redes de reparaciones y remanufactura en problemas de localización de instalaciones, formulado en un modelo de programación lineal entera mixta y resuelto con técnicas de Branch \& Bound. Por su parte, Jayaraman et al [23], plantearon un modelo general para el problema de distribución en la gestión del flujo de productos retornados, para un solo período. Propusieron además, una metodología de solución con técnicas heurísticas.

Gomes et al. [24], por su parte, proponen un modelo general para redes de logística inversa con retorno de productos e incertidumbre. A diferencia de Yongsheng et al. [22], Gomes et al. [24], sí consideran las capacidades de las instalaciones y analizan diferentes escenarios. 
Listes 0 . [4], propone un modelo de programación entera mixta estocástico para el análisis de redes de logística inversa de ciclo cerrado en actividades de remanufactura, con una función que considera costos e ingresos netos y busca minimizar el total de costos. En la función objetivo, hace una consideración de escenarios de demanda y retornos, y establece probabilidades de ocurrencia. Usa relajaciones lineales para solucionar el problema. Los resuelve finalmente, por técnicas de Branch and cut.

Figueiredo et al. [7], formulan un modelo de programación no lineal (MINLP), con una función de costos, estableciendo un modelo para una red de reciclaje con sistema de incentivos en el tema de la gestión de llantas fuera de uso. El modelo considera la capacidad de las plantas y múltiples períodos. Como método de solución, implementaron Multiplicadores de lagrange, bisección, series de fibonacci y una modificación del algoritmo de Teitz y Bart usado para encontrar la optima partición n. Pishvaee et al. [25], por su parte, plantean un robusto modelo de programación lineal entera mixta, para una red de circuito cerrado o abierto con incertidumbre, que considera la capacidad de las plantas, analizan diferentes escenarios. Sheu et al. [26], plantean un modelo de programación lineal multi-objetivo, con el fin de maximizar tanto el beneficio obtenido en la cadena de flujo directo, como en el proceso de logística inversa, en una cadena de ciclo cerrado. Asignan diferente peso a cada función objetivo, con el fin de distinguir los efectos de las funciones objetivo en un sistema de optimización que integra ambos procesos. Buscan encontrar un equilibrio entre los ingresos y los costos de ambos casos.

Schultmann et al. [27] plantean un modelo basado en el problema denominado symmetric capacited vehicle routing problem (SCVRP), con un depósito y un máximo tour de distancia. El objetivo es generar la asignación de un tour con mínimo costo, con $n+1$ nodos, con $n$ puntos de desmantelamiento, un deposito y $M$ tours. Utilizan técnicas heurísticas para solucionar el problema, alterando la función objetivo que minimiza la distancia, con la adición de parámetros que penalizan por exceder la máxima distancia y la máxima capacidad por tour. Resuelven el problema, implementando la búsqueda tabú y analizan los escenarios que varían la cantidad de puntos de desmantelamiento.

En la Tabla 1 se puede ver un resumen de los modelos, así como los métodos de solución empleados para evaluar las alternativas en el diseño de redes de logística inversa y localización de instalaciones.

\subsection{GESTIÓN DE INVENTARIOS EN REDES DE LOGÍSTICA INVERSA}

En segundo lugar, se encuentran las publicaciones que tratan el tema de gestión de inventarios. Estos artículos se pueden dividir en varios grupos: devoluciones comerciales, retornos durante la vida útil o al final de la vida útil del producto. 
Tabla 1. Problemas encontrados en la literatura de diseño de redes de logística inversa

\begin{tabular}{|c|c|c|c|}
\hline Autor & $\begin{array}{c}\text { Año } \\
\text { publicación }\end{array}$ & $\begin{array}{c}\text { Tipo de } \\
\text { problema }\end{array}$ & Método de solución \\
\hline \multicolumn{4}{|l|}{ MILP } \\
\hline Spengler et al. & 1997 & $\begin{array}{l}\text { DRPS } \\
\text { MSMPCFCP }\end{array}$ & $\begin{array}{l}\text { (LINDO) Descomposición de Benders. Caso } \\
\text { 2: (GAMS) }\end{array}$ \\
\hline Barros et al. & 1998 & MLCLP & Heurísticas (GAMS) \\
\hline Hu et al. & 2002 & MPMP & Lindo (linear, interactive and discrete optimizer) \\
\hline Jayaraman et al. & 2003 & FLP & $\begin{array}{l}\text { Heuristic concentration y heuristic expansion } \\
\text { (CPLEX) AMPL }\end{array}$ \\
\hline Schultmann et al. & 2006 & CLSC, SCVRP & Búsqueda tabú \\
\hline Lu y Bostel & 2007 & Two-level LP & Relajación langrangiana \\
\hline Gomes et al. & 2007 & CMPRLU & Técnicas de Branch \& Bound ( CPLEX) \\
\hline Yongsheng y Shouyang & 2008 & FLP & Técnicas de Branch \& Bound (Lingo) \\
\hline Cruz y Ertel & 2008 & FCFLP & Relajación langrangiana (SITATION) \\
\hline Srivastava & 2008 & MPME & GAMS \\
\hline Ortega & 2008 & STPNTCLP & Algoritmos genéticos, búsqueda dispersa \\
\hline Fan Wang y WeiHsu & 2009 & CLSC & Árbol de expansión-Algoritmos genéticos \\
\hline Pishvaee et al. & 2009 & UCLSC & ILOG CPLEX 10.1 \\
\hline \multicolumn{4}{|l|}{ SMIP } \\
\hline Listes & 2007 & $\begin{array}{l}\text { CLSC/ re- } \\
\text { manufacturing }\end{array}$ & branch-and-cut, integer L-shaped method \\
\hline \multicolumn{4}{|l|}{ MINLP } \\
\hline Figueiredo y Mayerle & 2008 & FLP & $\begin{array}{l}\text { Multiplicadores de lagrange, bisección, series } \\
\text { de fibonacci, Teitz and Bart algorithm }\end{array}$ \\
\hline \multicolumn{4}{|l|}{ MOP - MOMIP } \\
\hline Sheu et al. & 2004 & MODM & \\
\hline Du y Evans & 2007 & CFLP & Simplex dual-búsqueda dispersa \\
\hline Frota Neto et al. & 2008 & MODM & Frontera de Pareto \\
\hline Dehghanian y Mansour & 2009 & MODM, CFLP & Algoritmos genéticos-Frontera Pareto (Matlab) \\
\hline
\end{tabular}

MILP mixed integer linear programming, SMIP stochastic mixed integer programming, MINLP mixed integer non-linear programming, MOP multi-objective programming, MOMIP multi-objective mixed integer programming, SP location problem, FLP facility location and distribution problems, MLCLP capacited multi-level location problems, CFLP Capacitated Facility Location Problem, locationallocation problems, FCFLP Fixed Charge Facility Location Problem, MSMPCFCP Multistaged, multiproduct, capacited fixed charge problem, MPME multi-product, multi-echelon, DRPS dismantling and recycling planning system, MPMP multi-time-step, multi-type hazardous-waste, SCVRP symmetric capacited vehicle routing problem, Two-level LP two-level location problem, CLSC closedloop supply chain, UCLSC uncertainty closed-loop supply chain, CMPRLU capacitated multi-product reverse logistics network with uncertainty, STPNTCLP SingleTreatment Plant and Necessary Transfer Centers Location Problem, MODM multiple-objective decisionmaking. Fuente: Elaboración propia 
A menudo las devoluciones comerciales y los retornos se ven afectados por la incertidumbre sobre la cantidad que retornará y el tiempo cuando sucederá. La precisión en esta información, tendrá un impacto importante en la gestión de inventarios y en la disminución de costos logísticos. Es importante para las empresas, monitorear los retornos e investigar las razones por las cuales suceden. Al igual que en los problemas de localización y diseño de redes, se presentan modelos determinísticos y modelos estocásticos.

En su tesis doctoral, Van der Laan [28], analizó los efectos de la refabricación en el control del inventario. Los resultados de esta tesis fueron comprobados con datos reales de la refabricación de componentes de automóviles en Volkswagen.De Brito y Dekker [29], por su parte, exploran la validez de asumir el comportamiento de demanda y retornos en los modelos estocásticos para control de inventario, como homogéneos e independientes procesos de Poisson, analizando datos reales en flujos de retorno. Encuentran que no siempre esto es válido, y el investigador se debe preguntar si el retraso en el retorno sigue una distribución exponencial negativa.

Rubio S. [10], hace una clasificación de los sistemas de logística inversa y de los modelos cuantitativos empleados para su análisis, clasificándolos por las opciones de gestión para los productos recuperados (reutilización, reciclaje, refabricación, etc.). Complementa el estudio con un análisis cuantitativo de un sistema de gestión de inventarios, mediante un ejercicio de simulación dinámica, y hace un análisis de sensibilidad. De Brito M.P. [30], contribuyó a un mejor entendimiento de la logística inversa. En esta tesis, se reúnen todos los factores clave de la logística inversa y las relaciones que tienen entre sí, para luego proponer un marco teórico sobre la misma. De Brito y Van Der Laan [31], también investigan sobre cómo mejorar la toma de decisión en problemas de retornos y gestión del inventario, y analizan el impacto de la información imperfecta en problemas con retorno de productos en la gestión del inventario.

Lieckens et al. [32], plantearon un modelo general para redes de logística inversa, que combina modelos tradicionales con teoría de colas. Es un modelo de programación entera mixta no lineal que considera decisiones de localización, inventario y tiempos de entrega, con el objetivo de minimizar costos. Como método de solución, implementa algoritmos genéticos y una metodología de evolución diferencial.

Publicaciones más recientes, como Zerhouni et al. [33], consideraron dos sistemas de logística inversa donde los productos retornados están en buen estado, casi como nuevos. Para el primer ejemplo, la cantidad de productos retornados es independiente del flujo de la demanda. Ellos prueban que la política óptima está basada en el nivel de inventarios y se basan en parámetros como tasa de retorno, tasa de producción y, costos de producción, costos de retorno, entre otros. Para el segundo sistema, la cantidad de productos retornados, está relacionada fuertemente a la demanda, bajo la premisa de que una demanda satisfecha es inducida con cierta probabilidad a retornar un producto después de un tiempo determinado. Este problema es más complejo, sin embargo los autores logran un análisis adecuado para encontrar el nivel de inventarios óptimo. 
Gou et al. [34], plantean un modelo estocástico para encontrar el tamaño del lote optimo en la gestión de inventarios, con el objetivo de minimizar el costo promedio en la gestión de productos retornados a largo plazo, de múltiples puntos de recolección a un punto de productos retornados. Para solucionarlo, implementaron un modelo de simulación desarrollado en Matlab. Zhao et al. [35], plantearon un modelo que integra la gestión de inventarios y decisiones de transporte, de carácter estocástico, con el objetivo de minimizar los costos. En la metodología de solución, implementaron procesos de decisión de Markov. El modelo tiene en cuenta la capacidad de los vehículos de transporte así como varios períodos.

\subsection{PLANIFICACIÓN Y CONTROL DE ACTIVIDADES}

En tercer lugar, se encuentra la planificación y control de actividades. Este conjunto se puede subdividir en procesos de producción, recolección, recolección-distribución, desensamblado, etc. Algunas de las publicaciones encontradas sobre el tema son:

Krikke H. [36], trabajo acerca de la determinación de las estrategias de recuperación y el diseño de redes de logística inversa. Se pregunta cómo estructurar la recuperación de los flujos de retorno y dónde debe llevarse a cabo los procesos de recuperación y eliminación, una vez conocido el proceso de recuperación adecuado. En otro trabajo Krikke et al. [37], analiza las estrategias para un solo producto y para múltiples productos, y contiene un grupo de casos de estudio discutidos en detalle.

Kongar E. [38], hizo una revisión sobre diversas técnicas de decisión multi-criterio utilizadas para decidir la mejor técnica de desensamblaje en la recuperación de productos al final de su vida útil. Estos problemas, generalmente NP-completos, los resuelve mediante programación multiobjetivo y diversos meta-heurísticos.Kara et al. [39], han usado un modelo de simulación para evaluar los costos de una red de logística inversa para recolectar aparatos en el final de su vida útil e integra una actividad de desensamble en la red de logística inversa.

Kenne et al. [40], han usado un modelo de programación dinámica estocástica, y un algoritmo computacional basado en métodos numéricos, para planear y controlar la producción de un solo producto, en un sistema de remanufactura. Maneja tres tipos de inventario, el de productos manufacturados, remanufacturados, y retornados. El objetivo es proponer una política que minimice los costos globales.

De la Fuente et al. [41], examinan de manera conceptual las redes de ciclo cerrado, considerando nuevos lineamientos estratégicos y operacionales que incluyen información y coordinación de actividades.Con otros autores, De la fuente et al.[42],proponen un nuevo modelo metodológico llamado ERE-GIO aplicable a cadenas de reingeniería e integración de cadenas logísticas.

Mutha y Pokharel [43], diseñan una red de logística inversa para un caso de remanufactura, usando módulos para nuevos y viejos productos en un sistema con actividades de desmantelamiento, para 
remanufactura o venta en un mercado secundario. Considera los costos de transporte e inventarios y evalúa escenarios. Shi et al. [44], analizan un problema de planeación de la producción óptima para múltiples productos en un sistema cerrado con incertidumbre en demanda y retornos. Es un modelo de programación no lineal, resuelto con relajación langrangiana.

Otros autores que han probado modelos conceptuales $u$ otras técnicas, son: Trappey et al. [45], que desarrollan un modelo, usando mapas cognitivos y algoritmos genéticos para evaluar sistemas de identificación por radiofrecuencia en operaciones de logística inversa. El uso de mapas permitió expresar las relaciones causales entre los actores involucrados, e identificar los parámetros asociados; los algoritmos genéticos contribuyeron como soporte a las decisiones. Min et al. [46], desarrollan un modelo de programación no lineal entera mixta, para productos retornados, con el objetivo de determinar el número de puntos de acopio o recolección óptimo. El modelo es resuelto con algoritmos genéticos.

Lee y Dong [47], han desarrollado dos modelos para el diseño de una red de logística inversa de carácter dinámico, bajo incertidumbre. Un primer modelo propuesto es de tipo determinístico y el segundo estocástico. Han usado un algoritmo heurístico para la solución. Los resultados demostraron mayor eficiencia para el modelo estocástico.

González Torre et al. [48], analizan la implementación de prácticas de logística inversa en dos sentidos en colaboración con los proveedores y en colaboración con los clientes en el retorno de envases y embalaje. Chen et al. [49], motivados por las reglamentaciones gubernamentales, analizan un problema de reciclaje mediante un modelo de asignación de tráfico. Amini et al. [50], diseñan una operación de logística inversa para servicios de reparación, analizando los costos de inventario y los costos logísticos.

En revistas nacionales, se han encontrado publicaciones como la de Rodríguez y Vidal [51], que han propuesto un método heurístico para el control de inventarios, aplicado a una empresa productora de textos escolares, y encontraron mejores resultados para la compañía. Por su parte, Monroy y Ahumada [52], documentan y analizan algunos casos relevantes en el ámbito nacional que avanzan en la consolidación de redes de logística inversa.

\section{MODELO DE LOGÍSTICA INVERSA PARA LA GESTIÓN EFICIENTE DE LLANTAS Y NEUMÁTICOS FUERA DE USO}

A partir del análisis realizado sobre los problemas presentados en el diseño de redes de logística inversa y la recuperación y valorización de materiales o productos al final de su vida útil, se ha podido definir y caracterizar las diferentes alternativas para la gestión de llantas y neumáticos fuera de uso, en las ciudades de Pereira y Dosquebradas (Colombia). En la primera fase, se requiere diseñar la red de logística inversa, y tomar decisiones acerca de la financiación de la red. 
La Figura 3 ilustra una descripción del funcionamiento de la red de logística inversa, considerando los actores involucrados en la cadena. El conjunto de generadores de llantas y neumáticos fuera de uso, se ha clasificado por tipo de vehículo, agrupando los tipos de vehículo que requieren el mismo número de llantas, del mismo tamaño y peso. Se hizo una proyección anual para un horizonte de cinco años, sobre el crecimiento del parque automotor en la ciudades de Pereira y Dosquebradas, por tipo de vehículo, basándose en los datos históricos de 20 años del comportamiento del registro inicial de vehículos en las secretarias de tránsito de ambas ciudades, implementando el método de doble promedio móvil, que es una técnica de pronóstico para datos con tendencia [53]

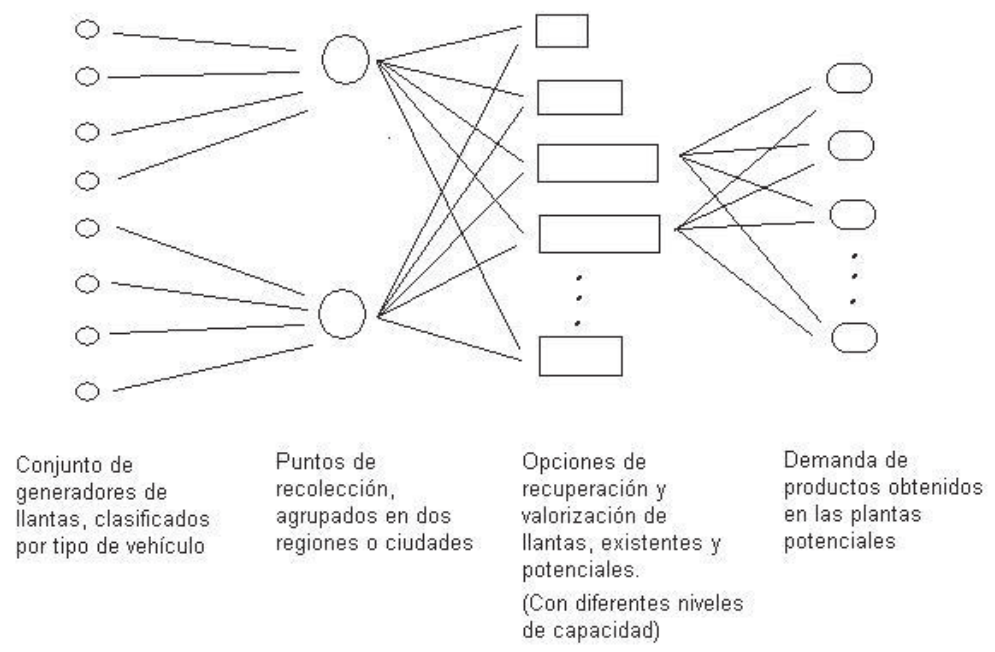

Figura 3. Red de logística inversa para la gestión de llantas y neumáticos fuera de uso.

Fuente: Elaboración propia.

De acuerdo con el crecimiento del parque automotor, se ha calculado la cantidad de llantas generadas en el mismo período en ambas ciudades, teniendo en cuenta el índice de generación de llantas usadas por tipo de vehículo, obtenido en un estudio realizado, sobre el diagnostico de esta problemática en la ciudad de Bogotá, para la secretaria de ambiente, de acuerdo, con los hábitos de uso y cambio de las llantas por parte de los usuarios.

Sin embargo, se requiere de estrategias y mecanismos de gestión, para hacer posible la recolección. Por este motivo, se han planteado dos escenarios posibles para evaluar: Escenario 1: Se recolecta el $100 \%$ de las llantas y neumáticos fuera de uso generados en cada período en las ciudades de Pereira y Dosquebradas. Escenario 2: Se recolecta el 20\% del total de llantas y neumáticos fuera de uso generados en el período 1 en cada ciudad, incrementado en 5\% cada período, de los cinco años siguientes, de acuerdo con las metas anuales de recolección establecidas en la Resolución 1457 de 2010, del Ministerio de Ambiente, vivienda y desarrollo territorial de Colombia. 
Las proyecciones generadas de llantas fuera de uso, se presentan en la Tabla 2, en unidades de $\mathrm{kg}$, producidas en ambas ciudades, de acuerdo con el peso del tipo de llanta que demanda cada tipo de vehículo.

Para la evaluación de las diferentes alternativas (procesos industriales o artesanales), se han determinado los costos de recolección y transporte por unidad de llanta y por kg de acuerdo con el tipo de llanta y las dimensiones y peso asociadas, proporcionales a las distancias que hay entre el lugar de origen y el destino de los mismos. Se ha establecido además, una ruta de recolección con frecuencia determinada, bajo la modalidad de negociación con los transportadores, hasta ocupar la capacidad máxima de los vehículos, para disminuir los costos logísticos.

Tabla 2. Cantidad de llantas fuera de uso generada

\begin{tabular}{|l|l|c|c|c|c|c|}
\hline \multirow{2}{*}{\begin{tabular}{c}
\multirow{2}{*}{$\begin{array}{c}\text { Tipo de } \\
\text { vehículo }\end{array}$} \\
\cline { 2 - 7 }
\end{tabular}} & Período actual & Año 1 & Año 2 & Año 3 & Año 4 & Año 5 \\
\hline AUTOMOVIL & $1,132,800.6$ & $1,190,179.8$ & $1,251,687.0$ & $1,313,168.4$ & $1,374,649.8$ & $1,436,157.0$ \\
\hline CAMION & $631,108.80$ & $648,950.40$ & $662,256.00$ & $675,864.00$ & $689,774.40$ & $702,777.60$ \\
\hline CAMIONETA & $757,770.00$ & $785,616.00$ & $810,030.00$ & $834,522.00$ & $859,014.00$ & $883,506.00$ \\
\hline CAMPERO & $432,734.40$ & $448,610.40$ & $462,621.60$ & $476,532.00$ & $490,543.20$ & $504,554.40$ \\
\hline MOTOS & $243,810.60$ & $271,609.80$ & $293,076.30$ & $314,536.20$ & $335,996.10$ & $357,456.00$ \\
\hline BUS & $313,513.20$ & $322,358.40$ & $328,746.60$ & $335,626.20$ & $342,014.40$ & $348,894.00$ \\
\hline BUSETA & $106,920.00$ & $111,600.00$ & $115,440.00$ & $119,160.00$ & $123,000.00$ & $128,760.00$ \\
\hline MICROBUS & $171,756.00$ & $179,556.00$ & $185,640.00$ & $191,724.00$ & $197,652.00$ & $203,736.00$ \\
\hline TOTAL & $3,790,413.6$ & $3,958,480.8$ & $4,109,497.5$ & $4,261,132.8$ & $4,412,643.9$ & $4,565,841.0$ \\
\hline
\end{tabular}

Por último, se han caracterizado las alternativas de gestión que existen en otras ciudades y sus costos logísticos asociados, así como las potenciales alternativas que podrían generarse en la Región, considerando los costos de apertura, asociados a la inversión de plantas industriales de trituración. Se considera diferenciado el costo fijo y variable por período de utilización de las plantas y se considera que una planta debe ser amortizada durante el horizonte contemplado. Las plantas potenciales tienen una capacidad determinada y diferente en cada alternativa por evaluar y se considera que se vende el 100\% de los productos obtenidos en la transformación de tipo industrial. Las alternativas de tipo artesanal representan opciones viables para la recuperación y aprovechamiento de neumáticos fuera de uso.

A partir del análisis de los modelos presentados en esta revisión, de la caracterización de diferentes alternativas para la Región y de la necesidad de evaluar cada alternativa, se presenta un modelo de programación lineal entera mixta (MILP), que busca evaluar las alternativas existentes, en términos de costo mínimo de operación y a su vez, las alternativas potenciales, maximizando el beneficio obtenido, expresado en términos de la rentabilidad económica obtenida de una posible 
valorización de llantas y neumáticos generados en ambas ciudades. Por último, se pretende evaluar las estrategias y los mecanismos de gestión para el establecimiento de la red de logística inversa alrededor del aprovechamiento de llantas y neumáticos fuera de uso, para las ciudades de Pereira y Dosquebradas. Los índices utilizados en el modelado son:

i= Conjunto de generadores clasificados por tipo de vehículo, localizados en ambas ciudades $(i=1,2,3,4,5)$

$j=$ Alternativas de recuperación de llantas (potenciales plantas de trituración) (j = 1,2,3)

$k=$ Alternativas de recuperación de llantas (existentes) ( $k=1,2)$

$m \quad=$ Alternativas de recuperación de neumáticos de tipo artesanal ( $m=1,2,3$ )

$0 \quad=$ Productos obtenidos en el proceso de trituración (o $=1,2,3,4,5)$

$h \quad=$ Período en años $(h=1,2,3,4,5)$

\section{Parámetros:}

$O_{\text {ih }}$ Cantidad de neumáticos obtenidos del tipo de vehículo i en el período h (Kg)

$A_{\text {ih }} \quad$ Cantidad de llantas obtenidas del tipo de vehículo i en el período h (Kg)

$B_{i j h}$ Costo de recolección y transporte de las llantas del tipo de vehículo i a la potencial planta j en el perío do $\mathrm{h}(\mathrm{s} / \mathrm{kg})$

$C_{i k h}$ Costo de recolección y transporte de las llantas del tipo de vehículo i a la planta existente $k$ en el perío do $\mathrm{h}(\mathrm{s} / \mathrm{kg})$

CAj Costos de apertura de una planta de tratamiento j (\$)

$C F_{i h}$ Costos fijos de la instalación j en el período $h(s)$

$C V_{\text {ih }}$ Costos variables de la instalación j en el período h $(\mathrm{s} / \mathrm{kg})$

$D_{j}$.h Capacidad máxima de la potencial planta de trituración j $(\mathrm{kg})$

$E_{k} \quad$ Capacidad máxima de la planta existente $\mathrm{k}(\mathrm{kg})$

$F_{j o h}$ Precio de venta del producto o obtenido en la plantaj en el período h $(\mathrm{s} / \mathrm{kg})$

$G_{i m h}$ Costos de recolección y transporte de los neumáticos del tipo de vehículo i al proyecto artesanal $m$ en el período $\mathrm{h}(\mathrm{s} / \mathrm{kg})$

$R_{m h}$ Precio de venta del neumático para la aplicación artesanal m en el período h $(\mathrm{s} / \mathrm{kg}$ )

$V_{j o} \quad$ Porcentaje de producto o obtenido en la plantaj por $\mathrm{kg}$ de llanta procesada.

\section{Variables de decisión:}

$W_{i k h}=$ Proporción de llantas del tipo de vehículo i, enviadas a la planta $\mathrm{k}$ en el período h (Kg)

$X_{i j h}=$ Proporción de llantas del tipo de vehículo i, enviadas a la planta j en el período h (kg)

$Y_{\text {imh }}=$ Cantidad de neumáticos del tipo de vehículo i, enviados al proceso artesanal $m$ en el período $h(\mathrm{Kg})$

$N_{j} \quad=$ Variable binaria que toma el valor 1 si la planta $j$, es abierta en el horizonte de tiempo contemplado y 0 en caso contrario $\{0,1\}$

$Z_{j h}=$ Variable binaria que toma el valor 1 si la planta $j$, es utilizada en el período hy 0 en caso contrario. $\{0,1\}$ 
$P_{k h}=$ Variable binaria que toma el valor 1 si la planta $k$ es asignada en el período h y 0 en caso contrario. $\{0,1\}$

\section{Función objetivo}

$$
\begin{aligned}
& \text { Maximizar } \sum_{i=1}^{5} \sum_{j=1}^{3} \sum_{h=1}^{5} \sum_{o=1}^{5} X_{i j h} A_{i h} v_{o j} F_{o j h}+\sum_{i=1}^{5} \sum_{m=1}^{3} \sum_{h=1}^{5} Y_{i m h} R_{m h} \\
& -\left[\sum_{j=1}^{3} C A_{j} N_{j}+\sum_{j=1}^{3} \sum_{h=1}^{5} C F_{j h} Z_{j h}+\sum_{i=1}^{5} \sum_{j=1}^{3} \sum_{h=1}^{5} C V_{j h} X_{i j h} A_{i h}+\sum_{i=1}^{5} \sum_{j=1}^{3} \sum_{h=1}^{5} B_{i j h} X_{i j h} A_{i h}\right. \\
& \left.+\sum_{i=1}^{5} \sum_{k=1}^{2} \sum_{h=1}^{5} C_{i k h} W_{i k h} A_{i h}+\sum_{i=1}^{5} \sum_{m=1}^{3} \sum_{h=1}^{5} Y_{i m h} G_{i m h}\right]
\end{aligned}
$$

La ecuación (2), maximiza el beneficio obtenido en la recuperación de las llantas fuera de uso, definido como la diferencia entre los ingresos (derivados de la venta de los productos, obtenidos de la trituración de llanta y venta de neumáticos), y la sumatoria de los costos de apertura de una potencial planta de tratamiento j, los costos fijos y variables de operación de la potencial planta en un período de cinco años, así como los costos de recolección y transporte de las llantas de los tipos de vehículo i a las plantas de tratamiento potencial $\mathrm{j}$ y/o a las plantas existentes $k$, y los costos de la gestión de neumáticos.

\section{S.A.}

$$
\begin{gathered}
\sum_{i=1}^{5} \sum_{j=1}^{3} \sum_{h=1}^{5} X_{i j h} \leq Z_{j h} \\
\sum_{h=1}^{5} Z_{j h} \leq 5 N_{j} \quad \forall j \\
\sum_{j=1}^{3} Z_{j h} \leq 1 \quad \forall h \\
\sum_{k=1}^{2} P_{k h} \leq 1 \quad \forall h \\
\sum_{j=1}^{3} X_{i j h}+\sum_{k=1}^{2} W_{i k h}=1 \quad \forall i, \forall h \\
\sum_{m=1}^{3} Y_{i m h} \leq Q_{i h} \quad \forall i, \forall h
\end{gathered}
$$




$$
\begin{gathered}
\sum_{i=1}^{5} X_{i j h} A_{i h} \leq D_{j} Z_{j h} \quad \forall j, \forall h \\
\sum_{i=1}^{5} W_{i k h} A_{i h} \leq E_{k} P_{k h} \quad \forall k, \forall h \\
\sum_{m=1}^{3} Y_{i m h} \geq Q_{i h} \quad \forall i, \forall h \\
Z_{j n h} \in\{0,1\} \quad \forall j, \forall n, \forall h \\
P_{k h} \in\{0,1\} \quad \forall k, \forall h \\
N_{j} \in\{0,1\} \forall j \\
X_{i j n h} \geq 0 \quad \forall i, \forall j, \forall n, \forall h \\
W_{i k h} \geq 0 \quad \forall i, \forall k, \forall h \\
Y_{i m h} \geq 0 \quad \forall i, \forall m, \forall h
\end{gathered}
$$

La restricción (3), establece que cuando se envíe una proporción de llantas a una planta j, la planta debe estar abierta, la restricción (4), activa los costos de apertura, estableciendo que si una planta j es utilizada en uno o varios períodos, quiere decir que la planta debe estar abierta y debe realizarse la inversión. Las restricciones (5) y (6), se refieren a que sólo una planta j y/o kdebe ser asignada para la gestión de llantas, en cada período de tiempo. La restricción (7) esta asociada al suministro y garantiza que todas las llantas generadas sean asignadas a alguna alternativa de recuperación; así mismo, la restricción (8) asegura que la cantidad enviada de neumáticos fuera de uso, es menor o igual a la cantidad generada para cada período. Las restricciones (9) y (10), no permiten que la proporción de llantas del tipo de vehículo i, enviadas a una potencial planta o existente, exceda la capacidad máxima de procesamiento de la planta. La restricción (1 1), establece la cantidad máxima que puede ser enviada por período, para las alternativas de neumáticos fuera de uso generado. El conjunto de restricciones (12) y (13), constituyen un problema combinatorio, garantizando que las proporciones enviadas sean variables con valores positivos entre 0 y 1 , y que las variables de decisión tomen valores binarios entre 0 y 1.

\section{Método de solución}

Para resolver el modelo, se ha implementado un modelador (GAMS 21.2), y un solver (Cplex), para encontrar las alternativas que maximizan el beneficio, enviando llantas fuera de uso tanto a alternativas potenciales, como existentes, con el fin de garantizar la gestión del 100\% de llantas y neumáticos fuera de uso generados. Debido a las dimensiones del problema de llantas fuera de uso, ha sido posible utilizar técnicas exactas (Branch and cut). 


\section{RESULTADOS Y ANÁLISIS}

Un primer escenario que considera la recolección del 100\% de llantas y neumáticos fuera de uso, permitió evaluar las alternativas para la obtención de beneficios, Y encontró una planta de capacidad menor como la alternativa más viable, con una inversión inicial de \$257.000.000. Las proyecciones financieras elegían la planta como una alternativa viable; no obstante el modelo propuesto, permitió evaluar la implementación de los costos logísticos de recolección y transporte, y asegurar su viabilidad. Un segundo escenario con un porcentaje de recolección gradual, iniciando con el $20 \%$ de la cantidad generada, permitió viabilizar la misma alternativa. Se pudo evidenciar que la capacidad óptima de las plantas, en un escenario de recolección del 100\%, requiere del apoyo de plantas existentes en otras ciudades, para su adecuada gestión, ya que implementar una planta de mayor capacidad no es viable para la cantidad de residuo que se genera. Sin embargo, como mecanismo de gestión más eficiente, se propone considerar un sistema de tarifas al consumidor y un ente gestor, en cuanto a la posibilidad de facilitar una recolección del 100\% de llantas fuera de uso, así como articular todos los actores de la cadena. La tarifa al consumidor estaría determinada por los costos logísticos por unidad de llanta.

Tabla 3.Resultados: Proporción de llantas del tipo de vehículo i enviadas a la planta j o k en el período h. Escenario del 100\% de recolección

\begin{tabular}{|c|c|c|c|c|c|}
\hline \multicolumn{6}{|c|}{ Función objetivo: 23.670.780.199,6962 } \\
\hline \multicolumn{6}{|c|}{ Alternativa seleccionada: Potencial Planta 2} \\
\hline \multirow{2}{*}{ Tipo de Ilanta } & \multicolumn{5}{|c|}{ Proporción } \\
\hline & Año 1 & Año 2 & Año 3 & Año 4 & Año 5 \\
\hline Moto & 1.000 & 1.000 & 1.000 & 1.000 & 1.000 \\
\hline Auto & 1.000 & 1.000 & 1.000 & 1.000 & 1.000 \\
\hline Camioneta & 1.000 & 1.000 & 1.000 & 1.000 & 1.000 \\
\hline Bus & 1.000 & 0.890 & 0.742 & 0.600 & 0.462 \\
\hline Busarticulado & 1.000 & & & & \\
\hline \multicolumn{6}{|c|}{ Alternativa seleccionada: Planta existente La-Tebaida } \\
\hline Bus & & 0.110 & 0.258 & 0.400 & 0.538 \\
\hline Bus articulado & & 1.000 & 1.000 & 1.000 & 1.000 \\
\hline
\end{tabular}

Fuente: Elaboración propia. Resultados obtenidos en GAMS

Los resultados obtenidos se presentan en la Tabla 3 y corresponden a las alternativas seleccionadas, así como las proporciones de llantas y neumáticos generados que deberían ser enviadas.

\section{CONCLUSIONES}

Se hizo una revisión del estado del arte de los problemas considerados en el diseño de redes de logística inversa, la cual dio como resultado un amplio espectro de posibilidades y variantes, acerca de los modelos de programación matemática y las técnicas de solución aplicadas. Se encontró que 
los modelos empleados han presentado una evolución, desde cuando se formularon modelos de programación lineal entera mixta, con la consideración de la capacidad de las instalaciones, pasando por modelos que consideraron el retorno de múltiples productos, y/o múltiples períodos (localización dinámica). Así mismo, aplicaciones en diferentes contextos, han dado lugar a nuevos planteamientos, y se encontraron modelos que consideran la incertidumbre en el retorno de productos (con carácter estocástico), así como análisis complejos que buscan tomar decisiones, bajo el análisis de otros criterios que influyen en las mejores alternativas por implementar, como es el caso de los modelos de programación multi-objetivo que involucran no sólo el análisis de costos, sino también el análisis costo-beneficio y el impacto ambiental y social, involucrando análisis de ciclo de vida (LCA), y análisis jerárquico. Por último, se han planteado modelos que integran el flujo directo e inverso en redes logísticas de circuito cerrado, maximizando beneficios económicos para las partes interesadas.

En cuanto a los métodos de solución empleados, no se encontró una constante. Los modelos genéricos han involucrado métodos exactos de búsqueda, con soluciones parciales que van acotando el problema, y permiten elaborar una solución completa, como Branch \& Bound. Otros han implementado algoritmos genéticos y otras técnicas heurísticas y meta-heurísticas. Los problemas de optimización multi-objetivo, donde las soluciones se califican con respecto de varias variables o criterios, y un problema tiene muchas soluciones posibles, han implementado la frontera eficiente de Pareto, para encontrar una solución. Otros autores han implementado Algoritmos de descomposición, dual simplex y búsqueda dispersa. Los métodos de solución en general, dependen del contexto, así como de la consideración o no de ciertos factores que influyen en el modelado del problema.

La aplicación a un caso real, permitió evaluar y determinar mejores alternativas de gestión acerca del aprovechamiento de productos fuera de uso. Para investigaciones futuras, se deja abierta la posibilidad de estudiar una mayor cantidad de alternativas potenciales de implementación, así como mejorar el modelo matemático con programación multi-objetivo, para hacer una comparación más eficiente de los costos y beneficios de las alternativas. Así mismo, al aumentar la complejidad del problema, será necesario implementar otras técnicas de solución del modelo presentado.

\section{REFERENCIAS BIBLIOGRÁFICAS}

[1] Pokharel S., and Mutha A., (2009). Perspectives in reverse logistics: A review. In: Resources, Conservation and Recycling, Vol. 53, pp. 175-182.

[2] Fleischmann M., Bloemhof-Ruwaard J.M., Dekker R., Van Der Laan E., Van Nunen Jo A.E.E and Van Wassenhove L., (1997). Quantitative models for reverse logistics: A review. In: European Journal of Operational Research, Vol. 103, pp. 1-17. 
[3] Bloemhof-Ruwaard J. M., Van Beek P., Hordijk L. and Van Wassenhove L. N., (1995). Interactions between operational research and environmental management. In: European Journal of Operational Research, Vol. 85, pp. 229-243.

[4] Listes O., (2007). A generic stochastic model for supply and return network design. In: Computers \& Operations Research, Vol. 34, pp. 417-442.

[5] Du F., and Evans G., (2008). A bi-objective reverse logistics network analysis for post-sale service. In: Computers \& Operations Research, Vol. 35, pp. 2617-2634.

[6] Dehghanian F., and Mansour S., (2009). Designing sustainable recovery network of end of life products using genetic algorithm. In: Resources, Conservation and Recycling, Vol. 53, pp. 559-570.

[7] Figueiredo J.N., and Mayerle S.F., (2008). Designing minimum cost recycling collection networks with required through put. In: Transportation Research Part E, Vol.44, pp. 731-752.

[8] Hesse M., and Rodrigue J.P., (2004).The transport geography of logistics and freight distribution. In: Journal of Transport Geography, Vol.12, pp. 171-184.

[9] Rogers D.S., and Tibben Lembke R.S., (1998). Going Backwards: Reverse Logistics Trends and Practices. Reverse Logistics Executive Council, Tesis Doctoral. Reno Center for Logistics Management. University of Nevada. 275 p.

[10] Rubio S., (2003). El sistema de logística inversa en la empresa: análisis y aplicaciones. Tesis Doctoral, Departamento de economía aplicada y organización de empresas, Universidad de Extremadura. Badajoz, 289 p.

[11] Ortega M., (2008). Utilización de métodos cuantitativos para el análisis de problemas de localización en logística inversa. Tesis Doctoral. Departamento de Ingeniería de Organización, Administración de Empresas y Estadística. Escuela Técnica Superior de Ingenieros Industriales. Universidad Politécnica de Madrid. Madrid, 276 p.

[12] Winston W.L., (2005). Investigación de operaciones. Aplicaciones y algoritmos. Cuarta edición. México D.F. International Thomson editores S.A.

[13] Thierry M., Salomon M., Van Nunen J., and Van Wassenhove L. (1995). Strategic Issues in Product Recovery Management. In: California Management review, Vol. 37, pp. 114-135.

[14] Spengler T., Piichert H., Penkuhn T., and Rentz O., (1997). Environmental integrated production and recycling management. In: European Journal of Operational Research, Vol. 97, pp. 308-326. 
[15] Barros A., Dekker R. and Scholten V., (1998). A two-level network for recycling sand: A case study. In: European Journal of Operational Research, Vol. 110, pp. 199-214.

[16] Cruz R., and Ertel J., (2009). Reverse logistics network design for the collection of End-of-Life Vehicles in Mexico. In: European Journal of Operational Research, Vol. 196, pp. 930-939.

[17] Srivastava S.K., (2008). Network design for reverse logistics. En: Omega, Vol. 36, pp. 535548.

[18] Fan Wang $H_{\text {., }}$ and Weihsu H., (2010). A closed-loop logistic model with a spanning-tree based genetic algorithm. In: Computers \& Operations Research, Vol. 37, pp. 376-389.

[19] Frota Neto J. Q., Bloemhof-ruwaard J. M., Van Nunen J.A.E.E., and Van Heck E., (2008). Designing and evaluating sustainable logistics networks. In: Int. J. Production Economics, Vol. 111, pp. 195-208.

[20] Hu T.L., Sheu J.B., and Huang K.H., (2002). A reverse logistics cost minimization model for the treatment of hazardous wastes. In: Transportation Research Part E, Vol. 38, pp. 457-473.

[21] Lu Z., and Bostel N. (2007). A facility location model for logistics systems including reverse flows: The case of remanufacturing activities. In: Computers \& Operations Research, Vol. 34, pp. 299-323.

[22] Yongsheng Z., and Shouyang W., (2008). Generic Model of Reverse Logistics Network Design. In: Journal of transportation systems engineering and information technology, Vol. 8(3), pp. 71-78.

[23] Jayaraman V., Patterson R.A., and Rolland E., (2003). The design of reverse distribution networks: Models and solution procedures. In: European journal of Operational Research, Vol. 150, pp. 128-149.

[24] Gomes M.I., Barbosa A.P. and Novais A.Q., (2007). An optimization model for the design of a capacitated multi-product reverse logistics network with uncertainty. In: European Journal of Operational Research, Vol. 179, pp. 1063-1077.

[25] Pishvaee M.S., Rabbani M., and Torabi S.A., (2011). A robust optimization approach to closed-loop supply chain network design under uncertainty. In: Applied Mathematical Modelling, Vol. 35, pp. 637-649.

[26] Sheu J.B., Chou Y.H., and Hu Ch., (2005). An integrated logistics operational model for green-supply chain management. In: Transportation Research Part E, Vol. 41, pp. 287-313. 
[27] Schultmann F., Zumkeller M., and Rentz O., (2006). Modeling reverse logistic tasks within closed-loop supply chains: An example from the automotive industry. In: European Journal of Operational Research, Vol. 171, pp. 1033-1050.

[28] Van Der Laan E., (1997). The effects of remanufacturing on inventory control. Tesis Doctoral. Rotterdam, Paises Bajos. Erasmus University Rotterdam.

[29] De Brito M.P. and Dekker R., (2003). Modelling product returns in inventory control: exploring the validity of general assumptions. In: Int. J. Production Economics 81-82, pp. 225-241.

[30] De Brito M.P., (2003). Managing Reverse Logistics or Reversing Logistics Management. Tesis Doctoral. Erasmus University Rotterdam. Erasmus Research institute of Management (ERIM).

[31] De Brito, M.P. and Van der Laan E.A. (2009). Inventory control with product returns: The impact of imperfect information. In: European Journal of Operational Research, Vol. 194, pp. 85-101.

[32] Lieckens K. and Vandaele N., (2005). Reverse logistics network design with stochastic lead times. En: Computers \& Operations Research.

[33] Zerhouni H., Gayon J.P. and Frein Y., (2010). Influence of dependency between demands and returns in a reverse logistics system. In: Int. J. Production Economics.

[34] Gou Q., Liang L., Huang Z., and Xu C., (2008). A joint inventory model for an open-loop reverse supply chain. En: Int. J. Production Economics, Vol. 116, pp. 28-42.

[35] Zhao Q., Chen S., Leung S., and Lai K.K., (2010). Integration of inventory and transportation decisions in a logistics system. In: Transportation Research Part E, Vol. 46, pp. 913-925.

[36] Krikke H., (1998). Recovery strategies and reverse logistic network design. Tesis Doctoral. Institute for Business Engineering and Technology Application (BETA); University of Twente. Países Bajos, 264 p.

[37] Krikke H., Van Harten A., and Schuur P., (1999). Business case roteb: recovery strategies for monitors. In: Computers \& Industrial Engineering, Vol. 364, pp. 739-757.

[38] Kongar E., (2003). Multiple Criteria Decision Making Techniques for End-Of-Life Product Recovery. Tesis Doctoral. Northeastern University. Boston, EEUU.

[39] Kara S., Rugrungruang F., Kaebernick $H_{\text {., }}$ (2007). Simulation modelling of reverse logistics networks. In: Int. J. Production Economics, Vol. 106, pp. 61-69. 
[40] Kenne J.P., Dejax P., and Gharbi A., (2009). Production planning of a hybrid manufacturing remanufacturing system under uncertainty within a closed loop supply chain. In: Int. J. Production Economics.

[41] De la Fuente M.V., Ros L., and Cardo M., (2008). Integrating Forward and Reverse Supply Chains: Application to a metal mechanic company. In: Int. J. Production Economics, Vol. 111 , pp. 782-792

[42] De la Fuente M.V., Ros L., and Ortiz A., (2010). Enterprise modelling methodology for forward and reverse supply chain flows integration. In: Computers in Industry, Vol. 61, pp. 702-710.

[43] Mutha A., and Pokharel S., (2009). Strategic network design for reverse logistics and remanufacturing using new and old product modules. In: Computers \& Industrial Engineering, Vol. 56, pp. 334-346

[44] Shi J., Zhang G., and Sha J., (201 1). Optimal production planning for a multi-product closed loop system with uncertain demand and return. In: Computers \& Operations Research, Vol. 38, pp. 641-650.

[45] Trappey J.C., Trappey A., and Wub Ch.V., (2010). Genetic algorithm dynamic performance evaluation for RFID reverse logistic management. In: Expert Systems with Applications, Vol. 37, pp. 7329-7335

[46] Min H., Ko H.J., and Ko Ch., (2006). A genetic algorithm approach to developing the multiechelon reverse logistics network for product returns. In: Omega, Vol. 34, pp. $56-69$

[47] Lee D.H., and Dong M., (2009). Dynamic network design for reverse logistics operations under uncertainty. In: Transportation Research Part E, Vol. 45, pp. 61-71.

[48] González Torre P.L., Adenso Díaz B., and Artibab H., (2004). Environmental and reverse logistics policies in European bottling and packaging firms. In: Int. J. Production Economics, Vol. 88, pp. 95-104.

[49] Chen H.K., Chou H.W., and Chiu Y.Ch., (2007). On the modeling and solution algorithm for the reverse logistics recycling flow equilibrium problem. In: Transportation Research Part C, Vol. 15, pp. 218-234.

[50] Amini M.M., Retzlaff Roberts D., and Bienstock C.C., (2005). Designing a reverse logistics operation for short cycle timerepair services. En: Int. J. Production Economics, Vol. 96, pp. 367-380. 
[51] Rodríguez J.A., and Vidal C.J., (2009). A heuristic method for the inventory control of short life-cycle products. En: Ingeniería y Competitividad, Vol. 11, No. 1, pp 37-55.

[52] Monroy N., and Ahumada M.C., (2006). Logística Reversa: Retos para la Ingeniería Industrial. In: Revista de Ingeniería, Vol. 23, pp. 23-33.

[53] Hanke J.E. and Wichern D.W., (2006). Pronósticos en los negocios. Cap. 4. 8 ed. México. Promedio móviles y métodos de suavizamiento. Pearson educación. 
